\title{
The log-transference and an average Gaussian
}

eye

\section{WF Harris*}

Optometric Science Research Group, Department of Optometry, and Department of Mathematics and Statistics, University of Johannesburg, PO Box 524, Auckland Park, 2006 South Africa

<wfh@na.rau.ac.za>

\begin{abstract}
Recent research on the logarithm of matrices is likely to have a significant impact on optometry. The purpose of this paper is to draw attention to this research and show application to the calculation of an average centred Gaussian eye in particular. The paper makes use of Cardoso's method to obtain an explicit expression for the logarithm of a transference. The arithmetic average of log-transferences leads to a transference which can be regarded as the transference of the average eye for the set. A numerical example is presented.
\end{abstract}

Most quantitative studies require, at some point, the calculation of an average. This fact has been the source of surprising difficulty in optometric research in particular. How does one calculate an average visual acuity or an average refractive error for example? These particular issues are discussed elsewhere ${ }^{1,2}$ The problem of calculating an average refractive error was solved as recently as 1983 by Keating; he showed that the average could be obtained as the arithmetic average of dioptric power matrices. ${ }^{3}$ But what about whole eyes? How can one calculate an average that in some sense averages the optical character of eyes in a set? This question has been the topic of recent work. ${ }^{4-9}$ While some unresolved issues remain it appears that the exponential-mean-logarithm of the transference may be the solution to the problem of the average eye. ${ }^{4}$ However, the trouble with the calculation of the exponential-mean-log transference, as described so far $^{4}$, is that it is purely mechanical; one has to use the matrix exponential and logarithm functions of specialist software like MATLAB. The functions are infinite series which make the mathematics anything but transparent and give little or no insight.

A doctoral thesis on the matrix logarithm, published in Portugal in 2003 by Cardoso ${ }^{10}$, now casts considerable light on the problem. The purpose of this paper is to draw attention to Cardoso's work which the author believes is destined to play a significant role in optometry and ophthalmology. More particularly the paper obtains an explicit exact expression for the logarithm of a transference and illustrates its use by applying it to the problem of the average Gaussian eye, that is, the eye that can be treated with traditional two-dimensional linear optics. Astigmatism is ignored and so are the effects of prismatic and decentred elements. The approach gives considerable insight and allows the calculation of the average Gaussian eye without the need for specialist computational software.

We begin by reviewing the concept of the transference of an optical system and the problem of calculating an average eye. We then use Cardoso's results ${ }^{10,11}$ to obtain an explicit ex- 
pression for the log-transference and for recovering a transference from its logarithm. Lastly we show, by means of a numerical example, application to the calculation of an average of two eyes. Our approach is mathematically informal. There are many technical mathematical issues which we shall ignore for the most part. The reader wishing to follow them up might consult the literature. ${ }^{4-11}$

\section{The transference}

The first-order optical nature of a centred Gaussian optical system is completely characterised by the transference ${ }^{12,13}$

$$
\mathbf{T}=\left(\begin{array}{ll}
A & B \\
C & D
\end{array}\right)
$$

of the system. $A$, the dilation, $B$, the disjugacy, $C$, the divergence, and $D$, the divarication, are the fundamental first-order optical properties of the system. (Terminology is discussed elsewhere. ${ }^{14}$ ) Other optical properties, called derived properties, of the system can be obtained from them. Perhaps the simplest example is the power of the system which is given by ${ }^{15}$

$F=-C$.

Another, when the system is an eye, is the corneal-plane refractive error which is given by ${ }^{16}$

$$
F_{0}=B^{-1} A \text {. }
$$

In fact when the system is an eye the dilation $A$ can be regarded as the ametropia of the eye. ${ }^{16}$ An eye with $A=0$ is emmetropic.

\section{An average eye}

At first glance one imagines one could calculate an average by simply averaging the transferences of the eyes in question. The average transference would then define an average eye. The strategy fails, however, because of symplecticity. A transference is necessarily symplectic, which means, in this case, that the transference has a unit determinant or, in other words, the fundamental properties are related by
$A D-C B=1$.

If one attempts to calculate an average in this naïve way of simply averaging the transferences one typically obtains an 'average eye' with a 'transference' whose determinant is generally not 1 . Formally such an average eye, then, cannot exist. (Nevertheless, despite being fundamentally unsound, such naïve averages can be adequate. This is an issue that is discussed further below.)

The solution to the problem appears to lie in the equation

$$
\widetilde{\mathbf{T}}=\exp \left(\frac{1}{N} \sum_{i=1}^{N} \log \mathbf{T}_{i}\right) .
$$

There is a set of $N$ eyes whose transferences are $\mathbf{T}_{n} \cdot \tilde{\mathbf{T}}$ is the exponential-mean-log-transference defined elsewhere. ${ }^{4}$ It is the transference of the average eye for the set. The exponential of a square matrix $\mathbf{M}$ is defined by the infinite series

$\mathbf{M}=\mathbf{I}+\mathbf{M}+\frac{1}{2 !} \mathbf{M}^{2}+\cdots+\frac{1}{n !} \mathbf{M}^{n}+\cdots$

where $\mathbf{I}$ is an identity matrix. Any square matrix $\mathbf{X}$ that satisfies

$\exp \mathbf{X}=\mathbf{M}$

is called a logarithm of $\mathbf{M}$. In general there are many logarithms of any particular matrix $\mathbf{M}$. However in cases of interest to us there is a unique real logarithm called the principal logarithm and written $\log \mathbf{M} . \log \mathbf{M}$ and $\exp \mathbf{M}$ are given in MATLAB by the functions logm and expm respectively. Given the transferences of $N$ eyes application of equation 5 to obtain an average eye is routine. Several numerical examples are presented elsewhere. ${ }^{4}$

Without specialist software like MATLAB one cannot calculate this average. Even with MATLAB the calculation is purely mechanical and gives little insight.

\section{From the transference to its logarithm}

Cardoso shows that $\log \mathbf{M}$ can be simplified to a polynomial in $\mathbf{M} .^{10,11}$ Applying his method 
to the transference we find that

$\log \mathbf{T}=f_{1}(1) \mathbf{I}+f_{2}(1)(\mathbf{I}-\mathbf{T})$.

In the interests of mathematical transparency this formula for a matrix logarithm is a vast improvement on an infinite series. To determine $f_{1}(1)$ and $f_{2}(1)$ we need the characteristic polynomial of the matrix $\mathbf{I}-\mathbf{T}$, namely $\operatorname{det}(\lambda \mathbf{I}-(\mathbf{I}$ - T)). It turns out to be

$\lambda^{2}-p \lambda+p$

where

$p=2(1-s)$

and $s$ is the semi-trace of $\mathbf{T}$ :

$s=\frac{1}{2} \operatorname{tr} \mathbf{T}$.

Following Cardoso's method we use expression 9 to write down

$f_{1}(1)=\int_{0}^{1} \frac{p u}{1-p u+p u^{2}} d u$

and

$f_{2}(1)=\int_{0}^{1} \frac{-1}{1-p u+p u^{2}} d u$.

Performing the integrations we obtain

$f_{1}(1)=\frac{2(1-s)}{\sqrt{1-s^{2}}} \tan ^{-1}\left(\frac{1-s}{\sqrt{1-s^{2}}}\right)$

and

$f_{2}(1)=-\frac{2}{\sqrt{1-s^{2}}} \tan ^{-1}\left(\frac{1-s}{\sqrt{1-s^{2}}}\right)$.

Substituting into equation 8 and simplifying we obtain

$\log \mathbf{T}=k(\mathbf{T}-s \mathbf{I})$

where

$$
k=\frac{2}{\sqrt{1-s^{2}}} \tan ^{-1}\left(\frac{1-s}{\sqrt{1-s^{2}}}\right) .
$$

Given a transference $\mathbf{T}$ we obtain $s$ using equation 11 and $k$ using equation $17 . \log \mathbf{T}$ is then given by equation 16 .

From the log-transference to the transference

In order to recover the transference from its logarithm we proceed as follows. We represent $\log \mathbf{T}$ by $\hat{\mathbf{T}}$ and then write

$$
\hat{\mathbf{T}}=\left(\begin{array}{ll}
\hat{A} & \hat{B} \\
\hat{C} & \hat{D}
\end{array}\right) .
$$

Its determinant is

$$
\hat{d}=\operatorname{det} \hat{\mathbf{T}} \text {. }
$$

Solving equation 16 we find

$$
\mathbf{T}=\exp \hat{\mathbf{T}}=\frac{1}{k} \hat{\mathbf{T}}+s \mathbf{I} .
$$

From equation 16 we obtain a result that simplifies to

$$
\hat{d}=k^{2}\left(1-s^{2}\right)
$$

Substituting from equation 17 and solving we obtain the semi-trace

$$
s=\frac{1-\tan ^{2} \sqrt{\hat{d} / 4}}{1+\tan ^{2} \sqrt{\hat{d} / 4}}
$$

of the transference.

It follows that the transference $\mathbf{T}$ can be calculated from its principal logarithm $\hat{\mathbf{T}}$ as follows: one obtains $\hat{\mathrm{d}}$ from $\hat{\mathbf{T}}$ using equation 19 and then $s$ using equation $21 ; k$ is obtained in turn by means of equation 17; and, finally, $\mathbf{T}$ is given by equation 20 .

\section{A numerical example}

In order to illustrate the use of the formulae we consider the problem of finding an average of the following two arbitrarily chosen eyes: $|40[0.003]| 10[0.015]$ and $|44[0.003]| 8[0.016]$. This notation is that used elsewhere. ${ }^{14}$ The first case represents a refracting surface or thin lens (the cornea) of power $40 \mathrm{D}$ followed in turn by a homogeneous gap (the aqueous) of reduced width $0.003 \mathrm{~m}$, a refracting surface or thin lens (the natural lens of the eye) of power $10 \mathrm{D}$ and a 
homogeneous gap (the vitreous) of reduced width $0.015 \mathrm{~m}$. The transferences of the two eyes are obtained in the usual manner: ${ }^{12,13}$

$\mathbf{T}_{1}=\left(\begin{array}{cc}1 & 0.015 \\ 0 & 1\end{array}\right)\left(\begin{array}{cc}1 & 0 \\ -10 & 1\end{array}\right)\left(\begin{array}{cc}1 & 0.003 \\ 0 & 1\end{array}\right)\left(\begin{array}{cc}1 & 0 \\ -40 & 1\end{array}\right)$

and

$\mathbf{T}_{2}=\left(\begin{array}{cc}1 & 0.016 \\ 0 & 1\end{array}\right)\left(\begin{array}{cc}1 & 0 \\ -8 & 1\end{array}\right)\left(\begin{array}{cc}1 & 0.003 \\ 0 & 1\end{array}\right)\left(\begin{array}{cc}1 & 0 \\ -44 & 1\end{array}\right)$.

(Notice application of equation 2 in the second and fourth matrices on the right-hand sides of these two equations.) The results are

$\mathbf{T}_{1}=\left(\begin{array}{cc}0.1480 & 0.0176 \\ -48.8000 & 0.9700\end{array}\right)$

and

$\mathbf{T}_{2}=\left(\begin{array}{cc}0.0529 & 0.0186 \\ -50.9440 & 0.9760\end{array}\right)$

Units are omitted. They are the same for all transferences here: the diagonal entries are unitless, the top-right entry is in metres and the bottom-left in dioptres.

As done before ${ }^{4}$ we could make use of the MATLAB functions logm and expm to calculate the average via equation 5 . Instead we accomplish the same thing by using the exact expressions given above for the matrix logarithm (equation 16) and the matrix exponential (equation 20).

For the first eye equation 11 gives $s_{1}=0.5590$ and equation $17 k_{1}=1.1790$. Equation 16 then gives the log-transference

$$
\log \mathbf{T}_{1}=k_{1}\left(\mathbf{T}_{1}-S \mathbf{I}\right)
$$

of the first eye, that is,

$\log \mathbf{T}_{1}=\left(\begin{array}{cc}-0.4846 & 0.0207 \\ -57.5369 & 0.4846\end{array}\right)$.

Similarly, for the second eye we find that $s_{2}=0.5144, k_{2}=1.2016$ and, hence,

$\log \mathbf{T}_{2}=\left(\begin{array}{cc}-0.5546 & 0.0224 \\ -61.2164 & 0.5546\end{array}\right)$.

The arithmetic average of these two logarithms is the average log-transference
$\tilde{\hat{\mathbf{T}}}=\frac{1}{2}\left(\log \mathbf{T}_{1}+\log \mathbf{T}_{2}\right)$,

that is,

$\tilde{\hat{\mathbf{T}}}=\left(\begin{array}{cc}-0.5196 & 0.0215 \\ -59.3766 & 0.5196\end{array}\right)$.

We now have to determine the transference of the average eye from this log-transference. From equation 19 we obtain the determinant of the average log-transference, $\hat{d}=1.0084$. Equation 21 then gives the semi-trace of the transference of the average eye, $\widetilde{s}=0.5367$. Equation 17 gives $\tilde{k}=1.1902$. Finally, from equation 20 we obtain

$\widetilde{\mathbf{T}}=\frac{1}{\tilde{k}} \tilde{\hat{\mathbf{T}}}+\widetilde{\boldsymbol{S}} \mathbf{I}$

or

$\widetilde{\mathbf{T}}=\left(\begin{array}{cc}0.1002 & 0.0181 \\ -49.8882 & 0.9733\end{array}\right)$,

the transference of the average eye.

From equation 3 we find that the eyes have (corneal-plane) refractive errors $8.43 \mathrm{D}$ and 2.84 $\mathrm{D}$ respectively and that the average eye has refractive error $5.54 \mathrm{D}$. We note that the refractive error of the average eye differs from the average of the refractive errors, namely 5.64 D.

It is interesting to note that if we calculate the average transference naïvely, that is, merely as the arithmetic average of the individual transferences we obtain

$\left(\begin{array}{cc}0.1004 & 0.0181 \\ -49.8720 & 0.9730\end{array}\right)$

which is close to the average $\widetilde{\mathbf{T}}$ obtained above. However, the determinant is 0.9996 which shows that the matrix is not symplectic and, so, cannot strictly be a transference.

\section{Discussion}

It is perhaps surprising that the logarithm of a transference can be expressed in so elegant a form as in equation 16. More importantly the form of the relationships now becomes more obvious. Insight is greatly enhanced. Potential routes to solving problems become easier to see. And so can potential sources of difficulty. For example one immediately sees from equation 17 that eyes with $s^{2}$ close to 1 and higher are likely 
to cause difficulty in the sorts of calculation described here. In practice, however, one does not expect problems because, as in the numerical example, eyes typically have $s$ close to $\frac{1}{2}$. In actual fact when $s=1$ one can show that $k=1$ and equation 16 simplifies to $\log \mathbf{T}=\mathbf{T}-\mathbf{I}$. When $\mathbf{s} \leq-1$ the equations break down; there is in fact no real logarithm. When $s=0$ one finds that $\log \mathbf{T}=\frac{\pi}{2} \mathbf{T}$.)

For many purposes an arithmetic average of transferences, rather than of their logarithms, might be adequate.(See the numerical example.) One expects that to be so for eyes that are similar although, at least for now, we have to be satisfied with a vague statement like this. Nevertheless an average based on an arithmetic average of transferences is fundamentally unsound and may have unexpected consequences. It is best avoided.

The analysis here can be extended in principle to $4 \times 4$ transferences and so allow for astigmatism. However the number of terms in Equation 8 increases and the mathematics becomes considerably messier.

We have here touched on the matrix logarithm only in its simplest application, perhaps, in optometry. In my view it is destined to make an impact on optometric research and, eventually, on optometric practice.

\section{Acknowledgements}

This paper is based on work supported under Grant Number 2053699 by the National Research Foundation. Much of the inspiration arises out of the work of Dr JR Cardoso of the Instituto Superior de Engenharia de Coimbra for whose interest and assistance I am particularly grateful. I also thank Drs RD van Gool and GE MacKenzie of the Optometric Science Research Group for continuing discussions.

\section{References}

1. Harris WF, Rubin A. Mean visual acuity. Ophthal Physiol Opt 199212392.

2. Saunders H. A method for determining the mean value of refractive errors. Br J Physiol Opt 1980 34 1-11.

3. Keating MP. On the use of matrices for the mean value of refractive errors. Ophthal Physiol Opt 1983 3 201-203.

4. Harris WF. The average eye. Ophthal Physiol Opt
200424 580-585.

5. Blendowske R. The average eye. Ophthal Physiol Opt 200525 459-460.

6. Harris WF. [The average eye.] Author's Reply. Ophthal Physiol Opt 200525 461-462.

7. van Gool RD, Harris WF. The concept of the average eye. (Abstract) Optom Vis Sci 200481 (12S) 163.

8. van Gool RD, Harris WF. The concept of the average eye. S Afr Optom 200564 38-43.

9. Harris WF, Cardoso JR. The exponential-mean-logtransference as a possible representation of the optical character of an average eye. Ophthal Physiol Opt 200525 in press

10. Cardoso JAR. Logaritmos de Matrizes: Aspectos Teóricos e Numéricos. Doctoral thesis, Department of Mathematics, University of Coimbra, Coimbra, Portugal, 2003.

11. Cardoso JR. An explicit formula for the matrix logarithm. S Afr Optom 200564 80-83.

12. Bamberg P, Sternberg S. A Course in Mathematics for Students of Physics: 1. Cambridge University Press, Cambridge, 1988 Ch 9.

13. Guillemin V, Sternberg S. Symplectic Techniques in Physics. Cambridge University Press, Cambridge, 1984.

14. Harris WF. Magnification, blur, and ray state at the retina for the general eye with and without a general optical instrument in front of it. 1. Distant objects. Optom Vis Sci 200178 888-900.

15. Harris WF. Dioptric power: its nature and its representation in three- and four-dimensional space. Optom Vis Sci 199774 349-366.

16. Harris WF. A unified paraxial approach to astigmatic optics. Optom Vis Sci 199976 480-499. 